\title{
Numerical Study of Chipless Tags for Radio-Frequency-Identification (RFID) Applications
}

\author{
Elif Çetin, M. Barış Şahin, Özgür Ergül \\ Department of Electrical and Electronics Engineering \\ Middle East Technical University, Ankara, Turkey \\ *corresponding author, E-mail: ozergul@metu.edu.tr
}

\begin{abstract}
We present a numerical investigation of effective chipless tags for radio-frequency-identification (RFID) applications. Chipless tags have been introduced recently as alternatives to standard tags with microchips. While they can significantly reduce the overall cost of RFID systems by eliminating microchips and procedures to mount them on tags, chipless tags bring new challenges, especially in terms of identification reliability. We focus on tag structures that consist of resonators and consider alternative scenarios to find out potential misidentification cases. We also present the robustness of resonator-type elements in terms of fabrication errors, as well as array strategies to significantly increase electromagnetic responses of tags at the cost of reduced compactness.
\end{abstract}

\section{Introduction}

Radio-frequency-identification (RFID) systems have become major tools in many applications involving the identification and tracking of living and nonliving objects, particularly in the areas of healthcare, manufacturing, transportation, and security [1,2]. In the state-of-art technology, RFID systems may not readily replace barcodes systems that are widely used for contactless identification with their diverse advantages. However, barcodes typically need lineof-sight, making their usage limited to those applications where this is possible, while RFID systems become attractive due to their much better performance without line-ofsight $[3,4]$. In addition, using radio waves instead of optical waves, RFID systems typically have longer read range. Recent advances in the low-cost inkjet printing technology $[5,6]$ have made RFID tags directly printable, similar to barcodes. On the other hand, microchips located on RFID tags restrict their usage due to additional costs, because of the existence of microchips and extra procedures to mount them on tags, in vast amounts of production [7]. For overcoming these issues, new RFID systems involving chipless tags have emerged as promising solutions in the literature. Eliminating microchips from tags can significantly reduce the cost of RFID systems [8], while it also leads to more suitable structures in terms of flexibility and recyclability. However, this brings more challenges in the design of suitable tags for reliability and effectiveness of identification and tracking.
Data can be encoded on chipless RFID tags either in time domain or in frequency domain [7,9]. Frequencycoded chipless RFID tags are commonly composed of planar resonators, while ID codes are encoded according to the presence (bit 1) and absence (bit 0) of resonators [10]. Particularly, arrays of u-shaped resonating elements, each corresponding to a single tag and having a distinct resonance frequency, are commonly used structures [11]. These resonators create peaks in the backscattered wave spectrum so that the radar cross section (RCS) can be used for detecting existing peaks and identify/distinguish the related tag [12]. Recently, other types of chipless tags, such as involving legible letter-type components [15], are introduced in the literature, while they often need special design procedures in order to be as effective as resonator-based tags.

In this work, frequency-coded chipless RFID tags are investigated in detail. We consider the performance of a single tag, and demonstrate particular cases of bit sequences, for which identification can be risky. We also present the response of a tag when fabrication errors that are typical in low-cost inkjet printing mechanisms are introduced. A major challenge in chipless RFID systems is to maintain high RCS levels, as well as good distinction of peaks from the base RCS, for readability. Since this can be difficult for a single tag, we also present different array strategies to arrange multiple identical tags.

A particular tag structure considered in this paper is shown in Fig. 1. It involves $10 \mathrm{u}$-shaped resonators [11]. Despite it provides a capacity of maximum $2^{10}-1=1023$ bits (considering that some configurations do not lead to satisfactory RCS responses), investigation of this structure provides the required information on the working principles of those involving larger numbers of bits. As mentioned above, existence and absence of a bit corresponds to 1 and 0 , respectively, leading to 10-bit IDs. Tags with different IDs have distinct backscattered RCS responses, which can be distinguished from each other so that the ID codes can be determined via measurement. In the following section, we briefly present the major parameters of the simulation environment to investigate tags and obtain their electromagnetic responses. Section 3 presents analysis of the chipless RFID tag structure in different perspectives, particularly considering different configurations of bits. In Section 4, we consider the impact of potential fabrication errors on the char- 


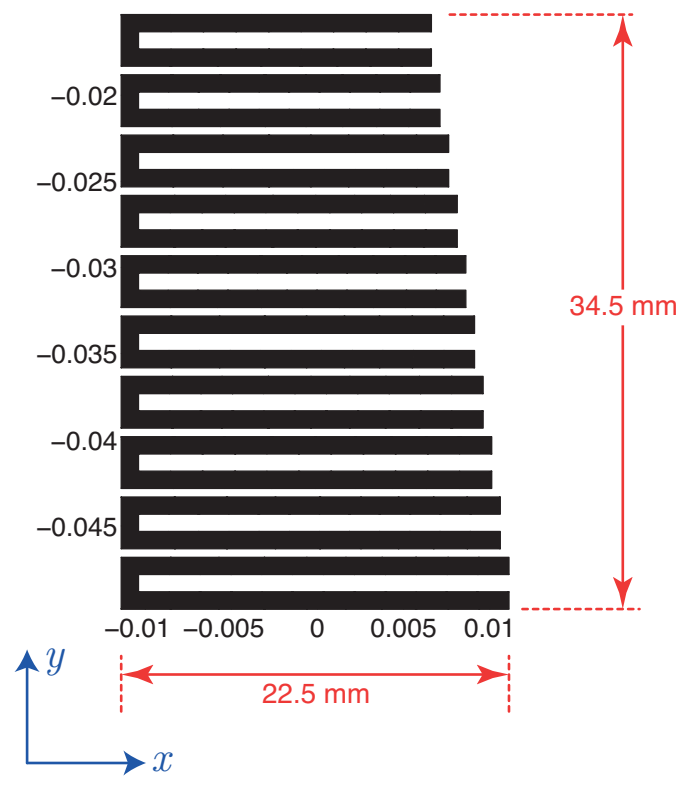

Figure 1: Single RFID tag structure that involves 10 resonators, each corresponding to a bit.

acteristics of RFID tags, followed by discussion of array strategies in Section 5 and concluding remarks in Section 6.

\section{Brief Description of the Simulation Environment}

Electromagnetic problems involving RFID tags are formulation with the electric-field integral equation (EFIE) in the frequency domain. Considering the frequency, surfaces are assumed to have zero thicknesses. EFIE and surfaces are discretized by using the Rao-Wilton-Glisson functions on triangles. The method of moments is used to construct matrix equations, which are solved iteratively using the fast multipole algorithm [14]. For RCS computations, tags are illuminated normally with a circularly polarized plane wave (except for the arrays in Section 5 that are also illuminated by a linearly polarized plane wave) in a range of frequencies. For the structure in Fig. 1, the frequency is sampled at $20 \mathrm{MHz}$ intervals from $3.0 \mathrm{GHz}$ to $5.0 \mathrm{GHz}$. Hence, for the complete characterization of this structure (considering 1023 different configurations), the total number of simulations is more than 100,000 . Once the coefficients expanding the electric current density is found, they are used to compute far-zone electric field intensity and RCS. Backscattered RCS is particularly considered as the signal reflected by a given tag.

\section{Analysis of the Chipless RFID Tag structure}

In this section, we consider the RFID tag structure in Fig. 1 in detail. We note that the tag covers an area of $22.5 \times 34.5 \mathrm{~mm}$ (approximately $7.76 \mathrm{~cm}^{2}$ ). The resonators are labelled from 1 (smallest resonator) to 10 (largest resonator). The overall RCS response of the tag with respect
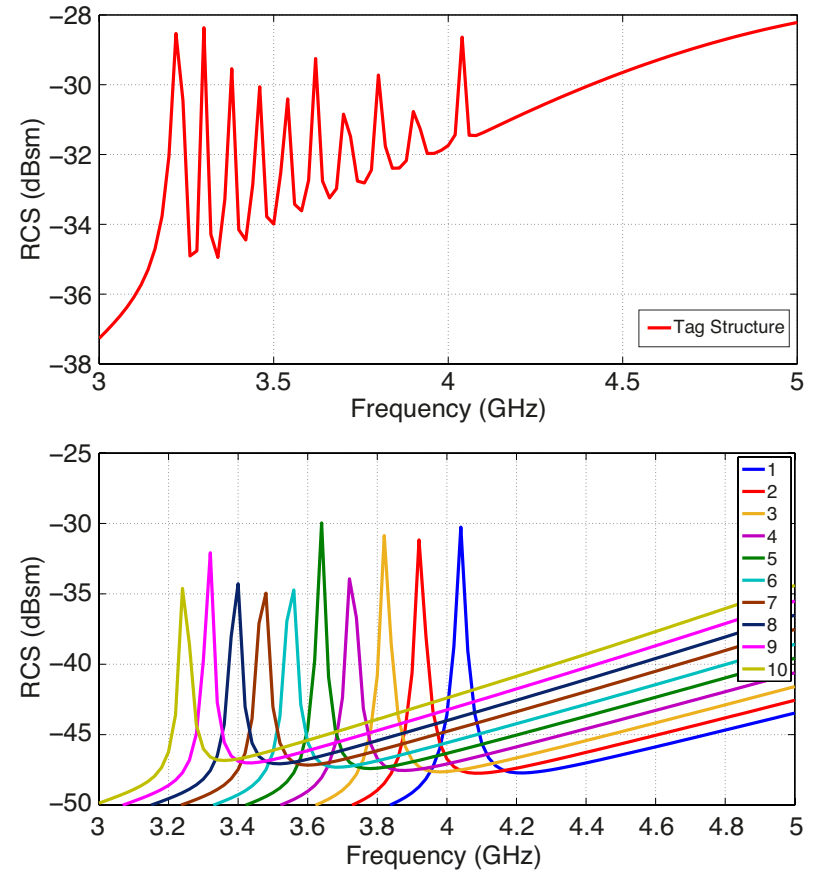

Figure 2: Electromagnetic response of the tag structure in Fig. 1, as well as the isolated characteristics of each resonator. The backscattered RCS is plotted with respect to frequency from $3.0 \mathrm{GHz}$ to $5.0 \mathrm{GHz}$.

to frequency is depicted in Fig. 2 (top), along with the RCS characteristics of each resonator alone (bottom). It can be observed that the resonance frequencies change in the 3.2$4.1 \mathrm{GHz}$ range. Combining the resonators has little effect on the resonance frequencies, while the amplitudes are affected due to coupling between the resonators. Electromagnetic coupling is also an important factor in the response of a tag when resonators are deleted, as discussed below.

\subsection{Single-Bit Deletions}

Fig. 3 presents the first set of RCS results involving singlebit deletions. In each case of ten different scenarios, one of the resonators is extracted, corresponding to the deletion of one bit. The backscattered RCS is plotted in the $[-38,-28] \mathrm{dB}$ range (also for all single-tag results below), while the modified tags are compared with the full tag. In all results, the deleted bit is clearly observed as a missing peak, while the coupling between resonators further leads to deteriorations in the resonances related to undeleted bits. This is particularly the case for the middle bits. Specifically, when the 4th, 5th, 6th, 7th, and 8th bits are deleted, the peak of the next bit is strongly affected with a reduced strength.

\subsection{Three-Bit Deletions}

Fig. 4 presents the second set, where three bits out of ten are deleted. In all five cases, bits 1 and 10 that correspond to the largest and the smallest resonators are deleted, while the third (deleted) bit is selected as 2, 5, 6, 7, and 9. First, 


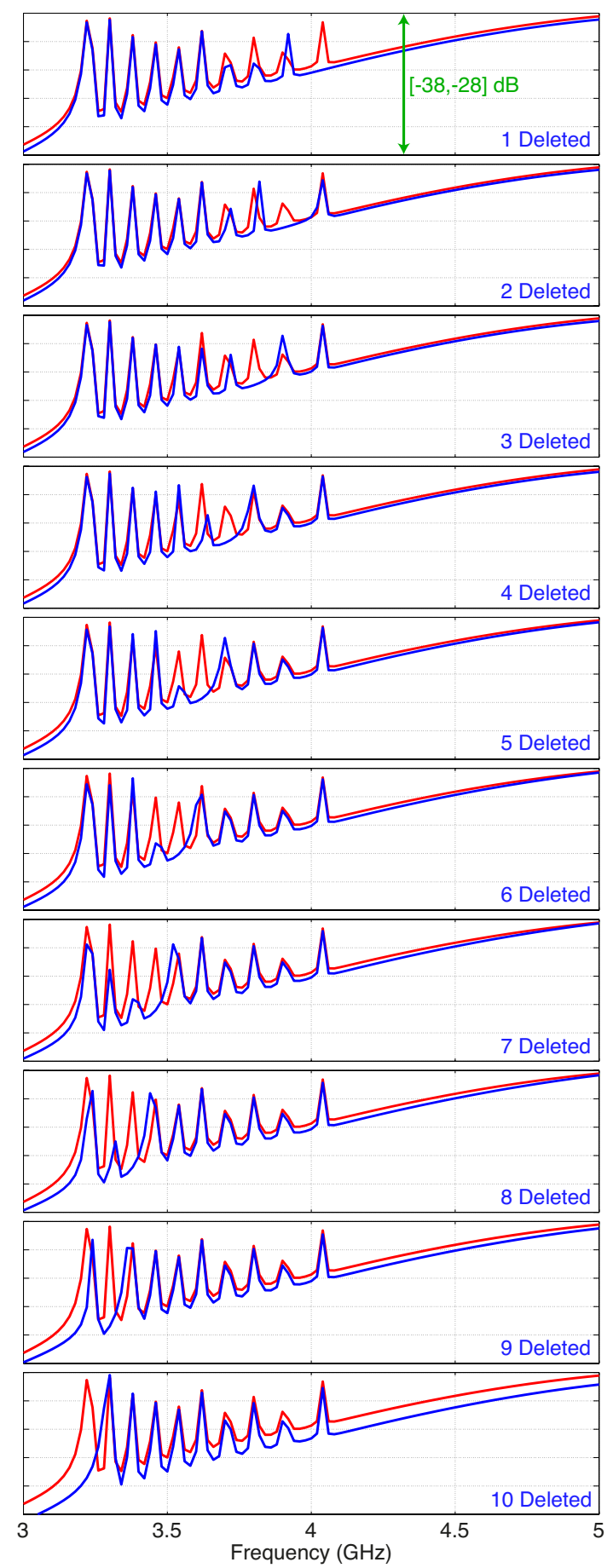

Figure 3: Electromagnetic responses of RFID tags that are obtained by deleting single bits from the overall structure in Fig. 1.

comparing with the results in Fig. 3, we note that the base RCS level is reduced due to the smaller numbers of remaining resonators. In addition, similar to the results in Fig. 3, deletion of a bit affects the next bit, while several peaks remain at their high positions. It is remarkable that, despite negative effects due to the coupling between resonators, the bits corresponding to the remaining resonators are clearly observed in all cases.

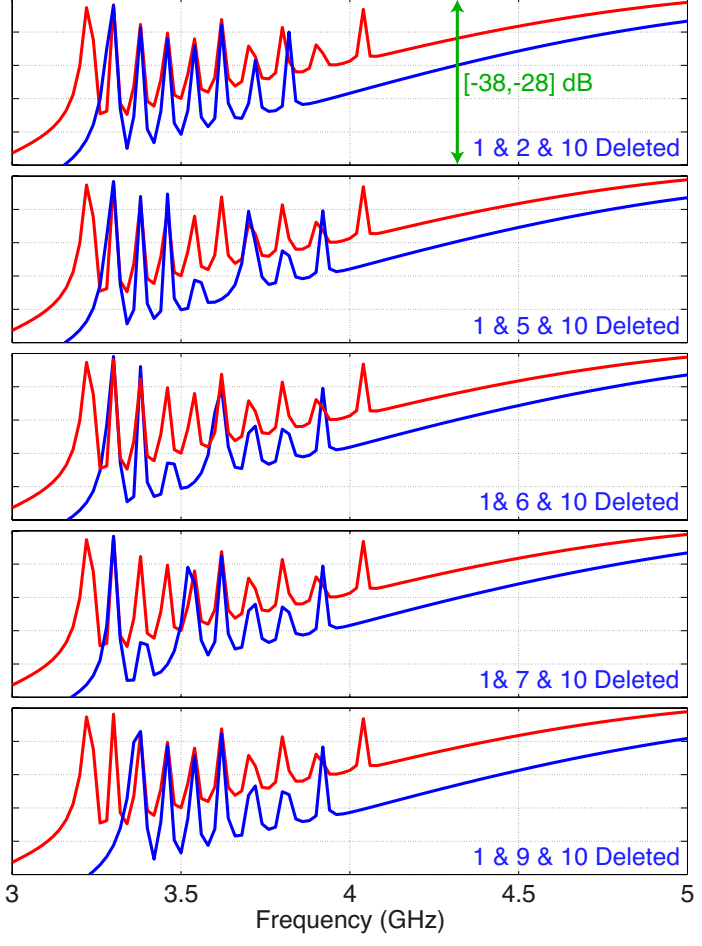

Figure 4: Electromagnetic responses of RFID tags that are obtained by deleting three bits from the overall structure in Fig. 1. The largest resonator is always missing.

\subsection{Consecutive Deletions}

Next, we consider a progressive deletion of bits in Fig. 5. In the first scenario, bits $2,3,4$, and 5 are deleted consecutively from a tag that already has missing bit 1 . In the second scenario, 7, 3, 9, and 1 are deleted consecutively from a tag with missing bit 5 . In the first sequence, it is obvious that the base RCS level decreases as more resonators are removed. Interestingly, the resonance related to the smallest undeleted bit seems to be affected significantly, while the remaining resonances keep their strong characteristics. In the second sequence, however, we observe both reduced value of the base RCS and remarkably decreased peaks. Once again, deleted bits strongly affect the resonances of the next peaks, leading to quite low levels, particularly when bits $1,3,5,7$, and 9 are deleted (so that bits $2,4,6,8$, and 10 are also affected).

\subsection{Block Deletions}

In Fig. 6, we consider the deletion of blocks of three bits, starting with $\{2,3,4\}$ to $\{8,9,10\}$. As the bit numbers increases, corresponding to larger resonators, the base RCS level decreases, while peak values are generally satisfied for undeleted bits. Once again, the most problematic bit seems to be the first undeleted one next to the deleted block. Fig. 7 presents similar results, where blocks of four and five bits are deleted. The reduced value of the base RCS is remarkable, especially when the block of bits $\{5,6,7,8,9\}$ are deleted, despite the remaining peaks are still recogniz- 

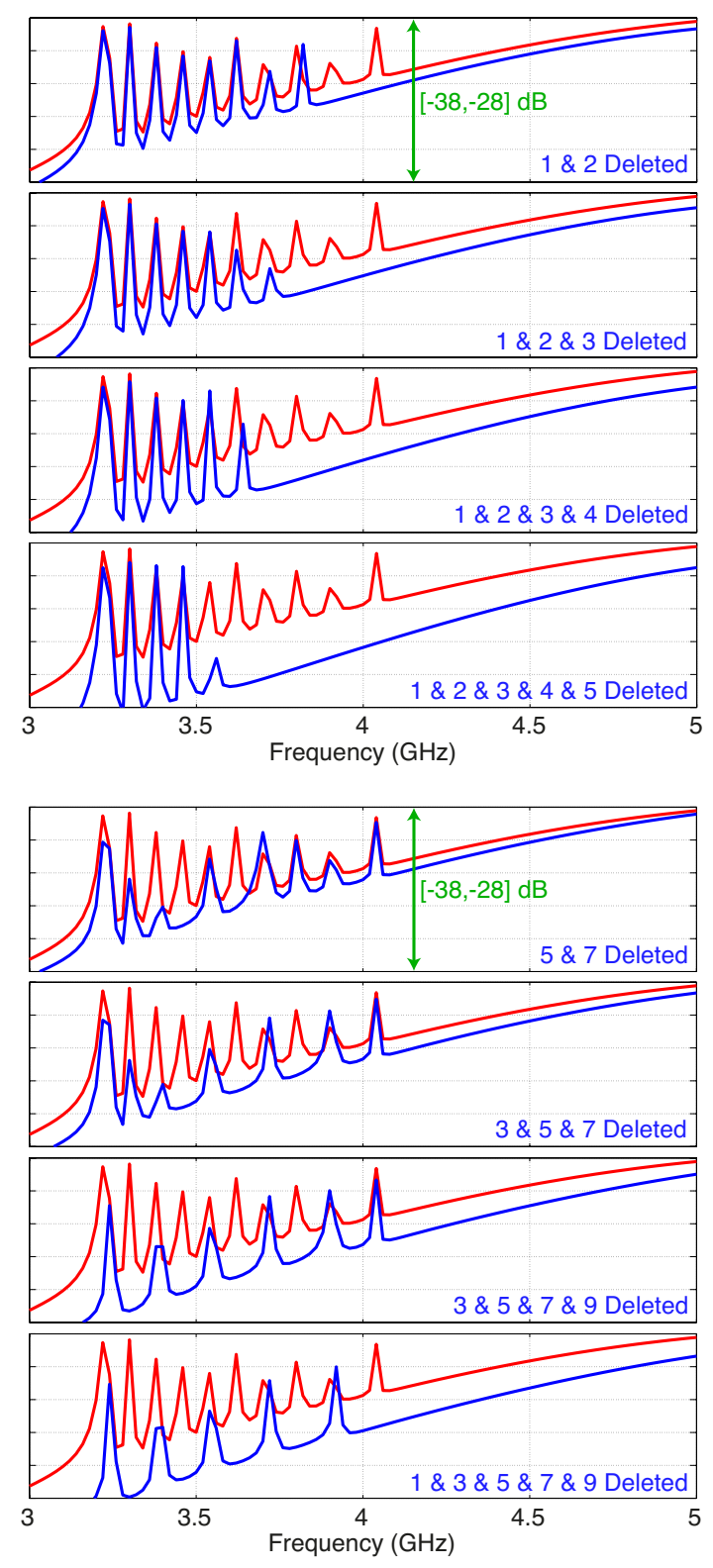

Figure 5: Electromagnetic responses of RFID tags that are obtained by deleting bits consecutively from the overall structure in Fig. 1.

able.

\subsection{Remarks on the Results of the Chipless RFID Tag}

Based on the results for different bit-deletion scenarios, some of which are demonstrated Figs. 3-7, some remarks are as follows.

- The chipless RFID tag structure based on resonators operates as designed by providing the required resonance peaks related to undeleted bits.

- Despite the remaining peaks remain recognizable, deletion of higher bits, corresponding to larger resonators, decreases the overall value of the RCS. Since most peaks can reach their original values, it is clear

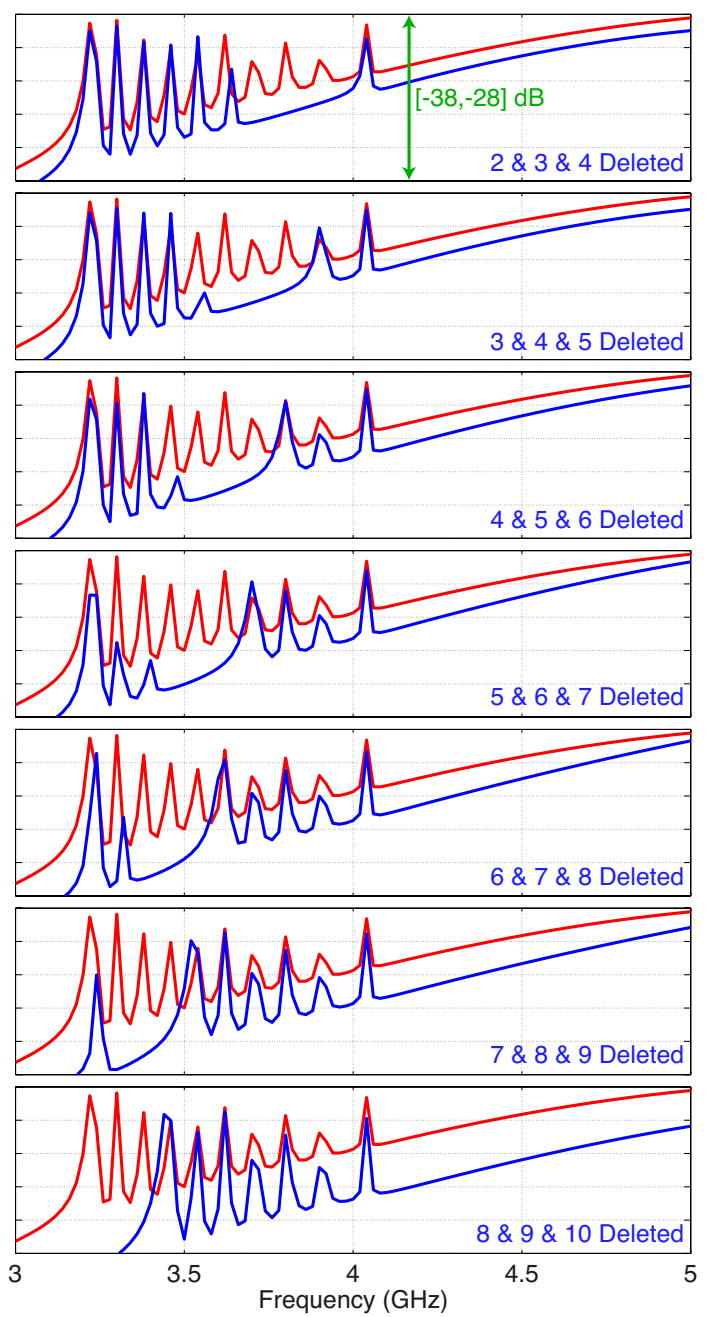

Figure 6: Electromagnetic responses of RFID tags that are obtained by deleting blocks of three bits from the overall structure in Fig. 1.

that the reduced base level is due to the decreased area of the tag when it is passive (between peaks).

- The most important issue seems to be related to undeleted bits near deleted bits. Specifically, when a bit $i<10$ is deleted and $(i+1)$ (corresponding to the next larger resonator) is undeleted, then the peak related to $(i+1)$ significantly deteriorates. This seems to be due to the unbalanced current distributions on the $(i+1)$ th resonator due to the absence of the $i$ th resonator. Interestingly, when $i>1$ is deleted, an undeleted $(i-1)$ is less affected (in fact, it may become even better), since this actually improves the balance of metals (considering resonator sizes) on the two sides of $(i-1)$. Cross investigations between diverse results in Figs. 1-7 further support these claims. As an example, for the 6th bit, the deletion of the block $\{3,4,5\}$ has disastrous effect (see Fig. 6), while a balanced deletion of bits 3, 5, and 7 (see Fig. 5) still keeps the 6 th peak at high levels. 


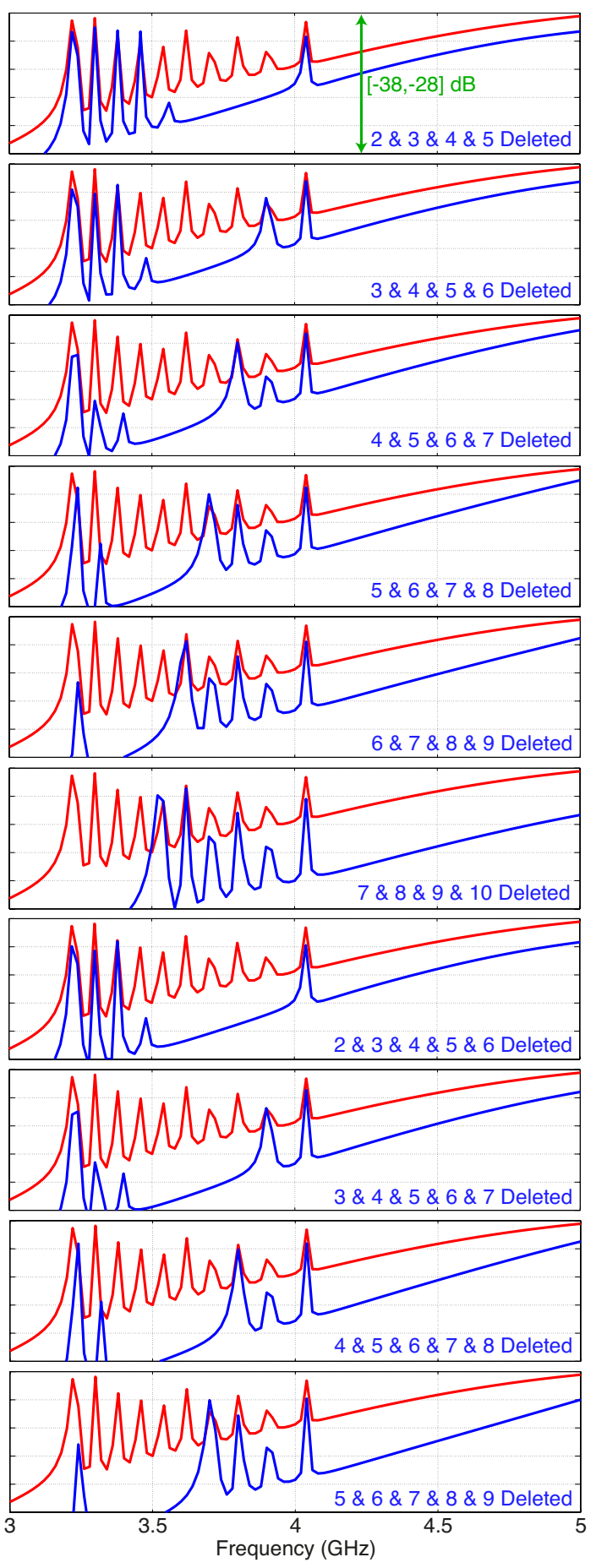

Figure 7: Electromagnetic responses of RFID tags that are obtained by deleting blocks of four or five bits from the overall structure in Fig. 1.

While, it may not be straightforward to categorize useless tags based on the geometry in Fig. 1, the results show that some identities are risky and may be eliminated directly from the RFID pool. For example, given a pair of IDs like

$$
\begin{aligned}
& {[\cdot, \cdot, \cdot, \cdot, 1,0, \cdot, \cdot, \cdot, \cdot \cdot]} \\
& {[\cdot, \cdot, \cdot, \cdot, 0,0, \cdot, \cdot, \cdot, \cdot],}
\end{aligned}
$$
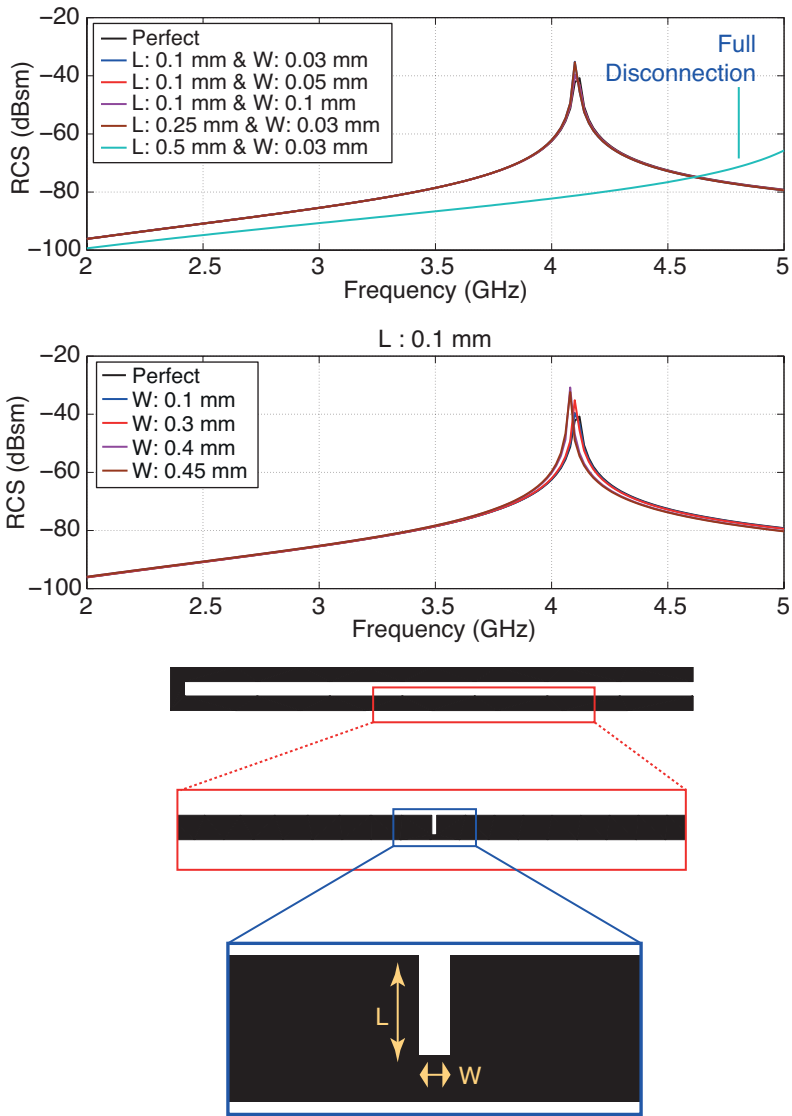

Figure 8: Electromagnetic response of a single resonator when there is a gap of width $W$ and length $L$ on one of the arms.

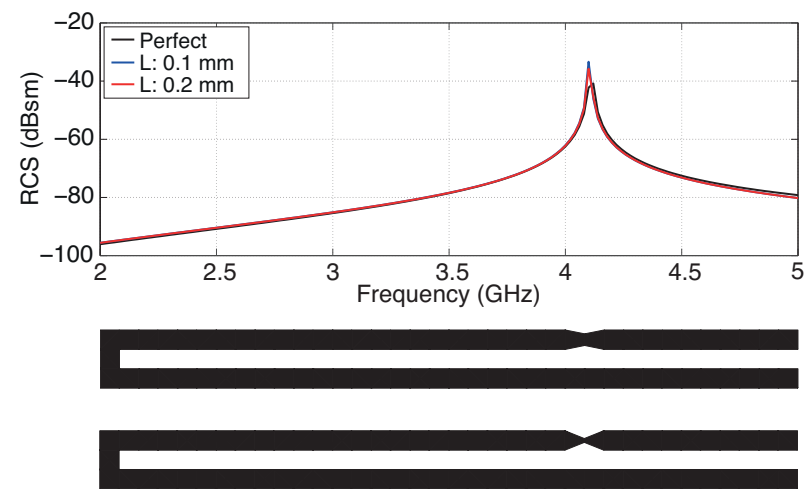

Figure 9: Electromagnetic response of a single resonator when there is thinning on one of the arms.

one of them can be eliminated to avoid misidentification. Alternatively, the distance between the resonators could be increased, while this would directly reduce the compactness and may not be preferred.

\section{Numerical Investigation of Potential Fabrication Errors}

In chipless RFID systems, tags are expected to be produced in large amounts so that they must be inexpensive as much 


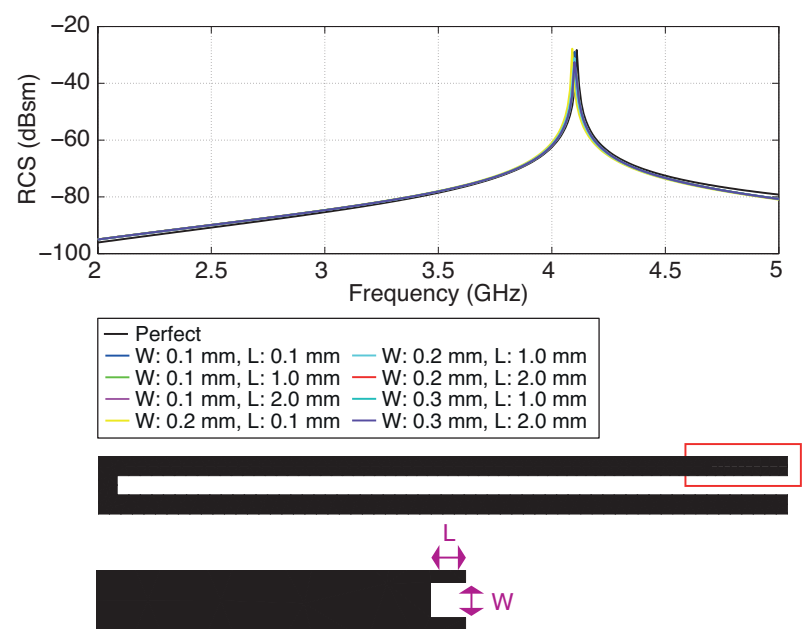

Figure 10: Electromagnetic response of a single resonator when there is crack on the tip of an arm.

as possible. In addition, most applications need flexible and environmentally friendly tags, making inkjet printing a suitable option for fabrication. On the other hand, samples produced by inkjet printing are prone to fabrication errors more than those produced via conventional techniques. Particularly, very low-cost inkjet setups are known to lead to resolution, conductivity, and other connectivity issues. Therefore, the designed RFID tags must be resistant to such fabrication errors. In fact, as shown below, tag structures, such as shown in Fig. 1, are quite robust from this perspective.

A main issue in low-cost inkjet printing is nonuniformity caused by limited printing resolution and unbalanced material (hence conductivity) distributions. The prints may be deformed, while physical movements, tensions, and bending may further cause cracks and gaps depending on the application. In Fig. 8, we consider the performance of a single resonator when there is a gap of width $W$ and length $L$ on one of the arms. Considering different lengths and widths, the resonance characteristics of the structure (RCS versus frequency) seem to be quite stable against such gaps. For example, only small frequency shifts occur as the width reaches $0.5 \mathrm{~mm}$. The length of the gap is also ineffective until it reaches $0.5 \mathrm{~mm}$ and full disconnection occurs. Fig. 9 presents similar results when there is a thinning ( $0.1 \mathrm{~mm}$ or $0.2 \mathrm{~mm}$ indentations from both sides) on one of the arms. The operation of the resonator is insensitive also to such deformations. Fig. 10 presents another set of results, where potential cracks that may occur on the tip of an arm are investigated. Different values are tested for the width of the crack as $W=0.1,0.2,0.3 \mathrm{~mm}$, as well as its length as $L=0.1,1.0,2.0 \mathrm{~mm}$. RCS with respect to frequency plots in Fig. 10 clearly show that the considered types of resonators are also stable against this kind of tip cracks.

In general, numerical results, some of which are shown in this paper, demonstrate that tag structures based on resonators, such as shown in Fig. 1, can be suitable to be fabricated via inkjet printing and similar techniques. Errors
Table 1: Sizes and general performances of the arrays in Fig. 11.

\begin{tabular}{cccc}
\hline & & $\mathrm{Q}[\mathrm{dB}]$ & $\mathrm{Q}[\mathrm{dB}]$ \\
Structure & Size $\left[\mathrm{cm}^{2}\right]$ & Linear & Circular \\
\hline Single Element & 7.8 & -29.8 & -29.6 \\
Array 1 & 84 & -12.7 & -12.6 \\
Array 2 & 131 & -7.62 & -8.00 \\
Array 3 & 119 & -8.93 & -8.34 \\
Array 4 & 49 & -22.7 & -22.7 \\
Array 5 & 49 & -22.6 & -22.6 \\
Array 6 & 66 & -22.3 & -22.3 \\
Array 7 & 66 & -22.5 & -22.2 \\
\hline
\end{tabular}

introduced during fabrications can be tolerated, until a total disconnection occurs such that the resonance path is completely damaged.

\section{Array Strategies}

In chipless RFID systems, the response of tags should be as strong as possible for reliable identification and tracking. On the other hand, even when using resonators, it can be difficult to increase RCS values. For this purpose, multiple tags can be arranged in array forms. While there can be many ways to construct arrays of tags, only some of them can provide the desired improvements. For demonstration, we consider seven different arrays involving alternative numbers of the structure in Fig. 1. The geometric configuration of the arrays, as well as the corresponding RCS values, for both linear (vertical) and circular polarizations, are depicted in Fig. 11. In each plot, RCS of a single structure is also shown for comparisons. While these plots provide important information on the RCS characteristics of the array, we further compare them quantitatively. For a selected interval of frequency containing all resonances, i.e., $[3.0,4.2] \mathrm{GHz}$ in our case, we compute

$$
Q=M+\sum_{i=1}^{10}\left(P_{i}-M\right)
$$

where $M$ is the mean value (over $\mathrm{dB}$ values) of the RCS in the interval, while $P_{i}$ for $i=1,2, \cdots, 10$ represents the peak values (in $\mathrm{dB}$ ) at resonances. A large value of $Q$ indicates good array performance with both large mean RCS $(M)$ and large peak-to-mean separation $\left(P_{i}-M\right)$. Table 1 lists the values of $Q$ for both linear (vertical) and circular polarizations, as well as the size of each array, in contrast to values for a single element. Comparing the results for linear polarization, performances of the first three arrays in comparison to other four (circular) are remarkable. Obviously, increasing the number of elements in the array improves the performance. Interestingly, these larger arrays (Arrays 1-3) perform better than circular ones (Arrays 4-7) even for circular polarization. Among circular arrays (Arrays 4-7), any of them can be used in terms of performance, while Arrays 4 and 5 come to forefront with their better 

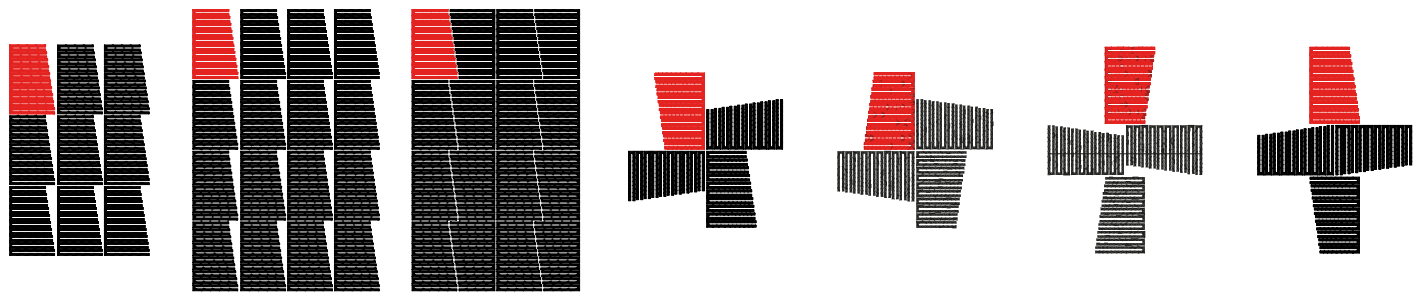

LINEAR POLARIZATION
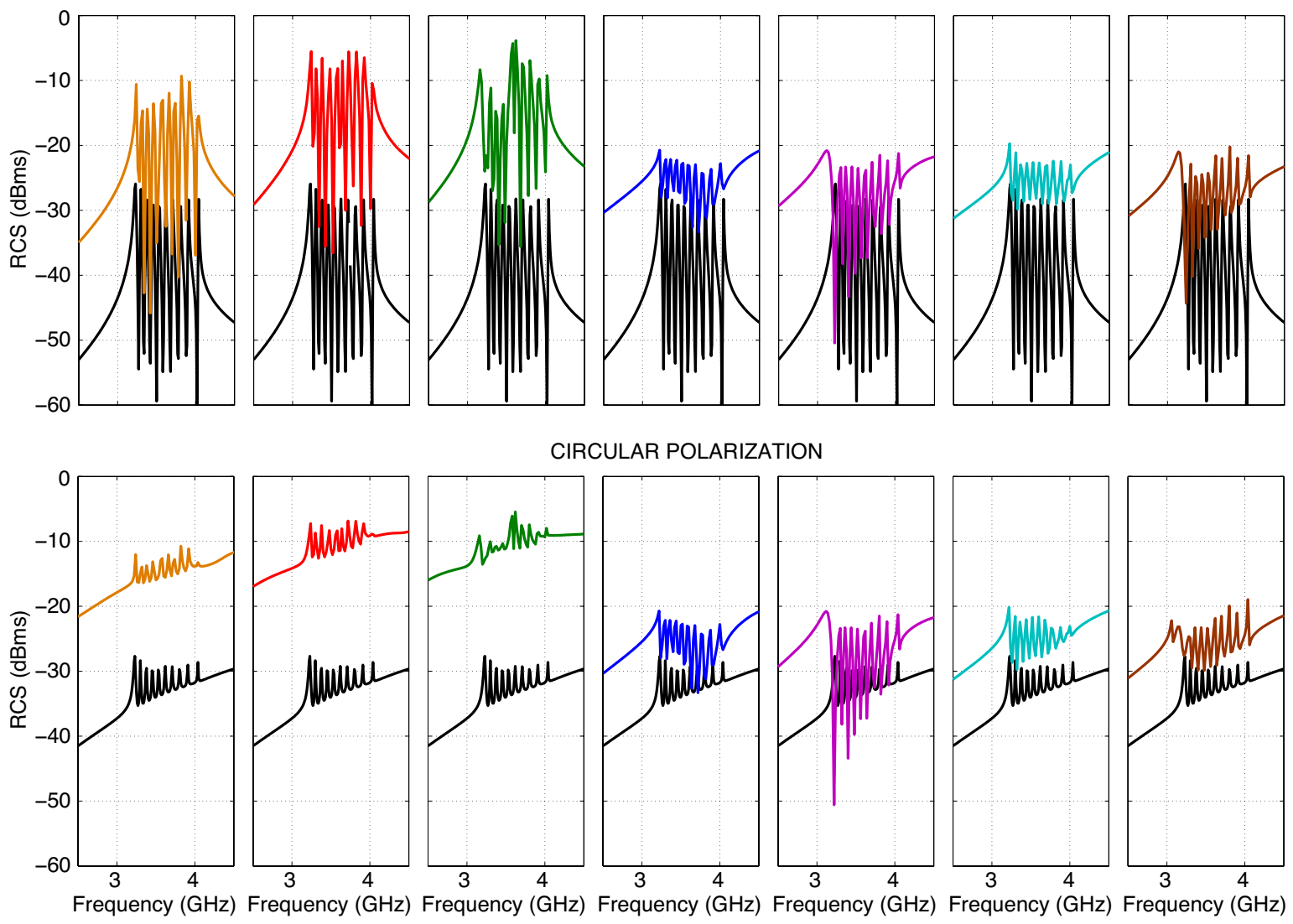

CIRCULAR POLARIZATION
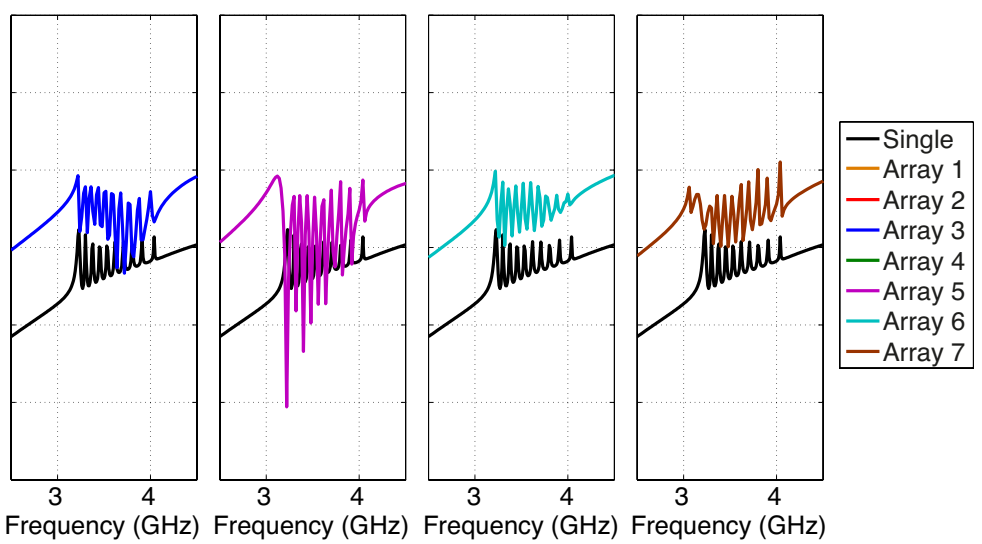

Figure 11: Different arrays involving alternative arrangements of multiple tags. RCS of each array is shown for both linear and circular polarizations, in comparison to those for a single tag.

compactness. Among Arrays 1-3, Fig. 11 shows that Array 3 does not provide good results in terms of the distinguishability of the peaks (even though this is not visible in $Q$ value). Arrays 1 and 2 seem to be very suitable in terms of base and peak RCS values, while their selection depends on the tradeoff between the performance and compactness.

\section{Concluding Remarks}

This study is devoted to numerical analysis of chipless RFID tags, which have been introduced as promising components of RFID systems due to their lower costs and better flexibility in comparison to tags with microchips. Chipless tags can also be more environmentally friendly since they do not contain microchips and they can be recycled easily. On the other hand, chipless tags bring new advantages, particularly in terms of readability and compactness. We present a detailed analysis of tags involving resonators and show their performance in alternative scenarios. This type structures seem to provide the required RCS levels, while they are also resistant to errors that may occur in their lowcost fabrications, e.g., via inkjet technology. These resonating structures can also be arranged in array forms such that RCS levels can significantly be increased at the cost of reduced compactness.

\section{Acknowledgement}

This work was supported by the Scientific and Technical Research Council of Turkey (TUBITAK) under the Research Grant 116 E871 and by the Turkish Academy of Sciences (TUBA).

\section{References}

[1] K. Finkenzeller, RFID Handbook: Fundamentals and Applications in Contactless Smart Cards and Identification, Wiley, New York, 2005. 
[2] S. Dey, J. Saha, N.C. Karmakar, Smart sensing: chipless RFID solutions for the internet of everything, $M i$ crow. Mag. 16: 26-39, 2015.

[3] S. Preradovic, N.C. Karmakar, Chipless RFID: Bar code of the future, Microw. Mag. 11: 87-97, 2010.

[4] S. Preradovic, I. Balbin, N.C. Karmakar, G. Swiegers, A novel chipless RFID system based on planar multiresonators for barcode replacement, Proc. IEEE Int. Conf. on RFID, pp. 289-296, 2008.

[5] T. Çiftçi, B. Karaosmanoğlu, Ö. Ergül, Low-cost inkjet antennas for RFID applications, IOP Conf. Ser.: Mater. Sci. Eng. 120: 1, 2016.

[6] F. Mutlu, C. Önol, B. Karaosmanoğlu, Ö. Ergül, Inkjet-printed cage-dipole antennas for radiofrequency applications, IET Microwaves, Antennas \& Propagation 11: 2016-2020, 2017.

[7] S. Preradovic, N.C. Karmakar, Fully printable chipless RFID tag, Advanced Radio Frequency Identification Design and Applications, Ed. S. Preradovic, InTech, 2011.

[8] H. Anam, A. Habib, S.I. Jafri, Y. Amin, H. Tenhunen, Directly printable frequency signatured chipless RFID tag for IoT applications, Radioengineering, 26: 139146, 2017.
[9] C. S. Hartmann, A global SAW ID tag with large data capacity, Proc. IEEE Ultrasonics Symp., pp. 65-69, 2002.

[10] S. Preradovic, I. Balbin, N.C. Karmakar, G.F. Swiegers, Multiresonator-based chipless RFID system for low-cost item tracking, IEEE Trans. Microw. Theory Tech., 57: 1411-1141, 2009.

[11] A. Vena, E. Perret, S. Tedjini, Chipless RFID tag using hybrid coding technique, IEEE Trans. Microw. Theory Tech. 59: 3356-3364, 2011.

[12] J. Havlicek, M. Svanda, J. Machac, M. Polivka, Improvement of reading performance of frequency domain chipless RFID transponders, Radioengineering, 25: 219-229, 2016.

[15] M. A. Demir, F. Mutlu, Ö. Ergül, Design of highly distinguishable letters for inkjet-printed chipless RFID tags, IEEE-APS Topical Conf. on Antennas and Propagation in Wireless Communications (IEEE APWC), pp. 783-786, 2018.

[14] Ö. Ergül, L. Gürel, The Multilevel Fast Multipole Algorithm (MLFMA) for Solving Large-Scale Computational Electromagnetics Problems, WileyIEEE, 2014. 